21st Particles and Nuclei International Conference (PANIC 2017)

International Journal of Modern Physics: Conference Series

Vol. 46 (2018) 1860085 (4 pages)

(C) The Author(s)

DOI: $10.1142 / \mathrm{S} 2010194518600856$

\title{
Determination of the Strong Coupling Constant $\alpha_{\mathrm{s}}$ in Multijet Production with the ATLAS Detector at the LHC
}

\author{
Javier Llorente (on behalf of the ATLAS Collaboration) \\ Institute of High Energy Physics, Chinese Academy of Sciences, China \\ javier.llorente.merino@cern.ch
}

Published 3 May 2018

\begin{abstract}
A measurement of transverse energy-energy correlations and its asymmetry in $p p$ collisions recorded by the ATLAS detector at the LHC at $\sqrt{s}=8 \mathrm{TeV}$ is presented. The results are used to determine the strong coupling constant $\alpha_{\mathrm{S}}\left(m_{Z}\right)$. A global fit to the transverse energy-energy correlation distributions yields $\alpha_{\mathrm{s}}\left(m_{Z}\right)=0.1162 \pm$ 0.0011 (exp. $)_{-0.0070}^{+0.0084}$ (theo.), while a global fit to the corresponding asymmetry distributions yields a value of $\alpha_{\mathrm{s}}\left(m_{Z}\right)=0.1196 \pm 0.0013$ (exp. $)_{-0.0045}^{+0.0075}$ (theo.).
\end{abstract}

\section{Introduction}

Energy-energy correlations were proposed as a precision test of Quantum Chromodynamics (QCD) in $e^{+} e^{-}$annihilation [1]. This observable, which exploits the phenomenon of azimuthal decorrelations, was further adapted to hadron-hadron collisions [2], and its next-to-leading order (NLO) corrections were calculated [3]. The transverse energy-energy correlation function (TEEC) can be calculated in a multijet event by considering the angles in the transverse plane $\phi_{i j}$ for all pairs of jets $(i, j)$, weighted with the normalised products of their transverse energies, i.e.

$$
\frac{1}{\sigma} \frac{\mathrm{d} \Sigma}{\mathrm{d} \cos \phi} \equiv \frac{1}{N} \sum_{A=1}^{N} \sum_{i j} \frac{E_{\mathrm{T} i}^{A} E_{\mathrm{T} j}^{A}}{\left(\sum_{k} E_{\mathrm{T} k}^{A}\right)^{2}} \delta\left(\cos \phi-\cos \phi_{i j}\right),
$$

where the index $A$ runs over all events in the sample, $i, j$ and $k$ are jet indices and $\delta(x)$ is the Dirac delta function, which ensures $\phi=\phi_{i j}$, the relative azimuth for each pair $(i, j)$. It is also customary to define the asymmetry on the TEEC function (ATEEC) as

$$
\left.\frac{1}{\sigma} \frac{\mathrm{d} \Sigma^{\operatorname{asym}}}{\mathrm{d} \cos \phi} \equiv \frac{1}{\sigma} \frac{\mathrm{d} \Sigma}{\mathrm{d} \cos \phi}\right|_{\phi}-\left.\frac{1}{\sigma} \frac{\mathrm{d} \Sigma}{\mathrm{d} \cos \phi}\right|_{\pi-\phi} .
$$

The dependence of the TEEC and ATEEC on the strong coupling constant, $\alpha_{s}$, and their mild dependence with other experimental and theoretical parameters make

This is an Open Access article published by World Scientific Publishing Company. It is distributed under the terms of the Creative Commons Attribution 4.0 (CC-BY) License. Further distribution of this work is permitted, provided the original work is properly cited. 


\section{J. Llorente}

them ideal for a determination of the strong coupling constant. The ATLAS Collaboration at the LHC [4] has recently presented a measurement of the TEEC and ATEEC functions [5].

\section{Measured distributions}

The TEEC and ATEEC functions are measured in six bins [5] of the scalar sum of the transverse momentum of the two leading jets, $H_{\mathrm{T} 2}=p_{\mathrm{T} 1}+p_{\mathrm{T} 2}$. All distributions are corrected for detector effects using a Bayesian iterative procedure [6]. Figure 1 shows the unfolded TEEC and ATEEC distributions for the highest $H_{\mathrm{T} 2}$ bin, $H_{\mathrm{T} 2}>$ $1400 \mathrm{GeV}$, together with the MC expectations from Pythia [7], Sherpa [8] and Herwig $++[9]$. The TEEC distribution shows two peaks in the endpoints of the distribution, $\cos \phi \sim \pm 1$. The first one, at $\cos \phi \sim-1$, is due to the back-to-back configuration of dijet events, where one jet recoils against the other one. The second one, at $\cos \phi \sim+1$, is due to self-correlations of one jet with itself.
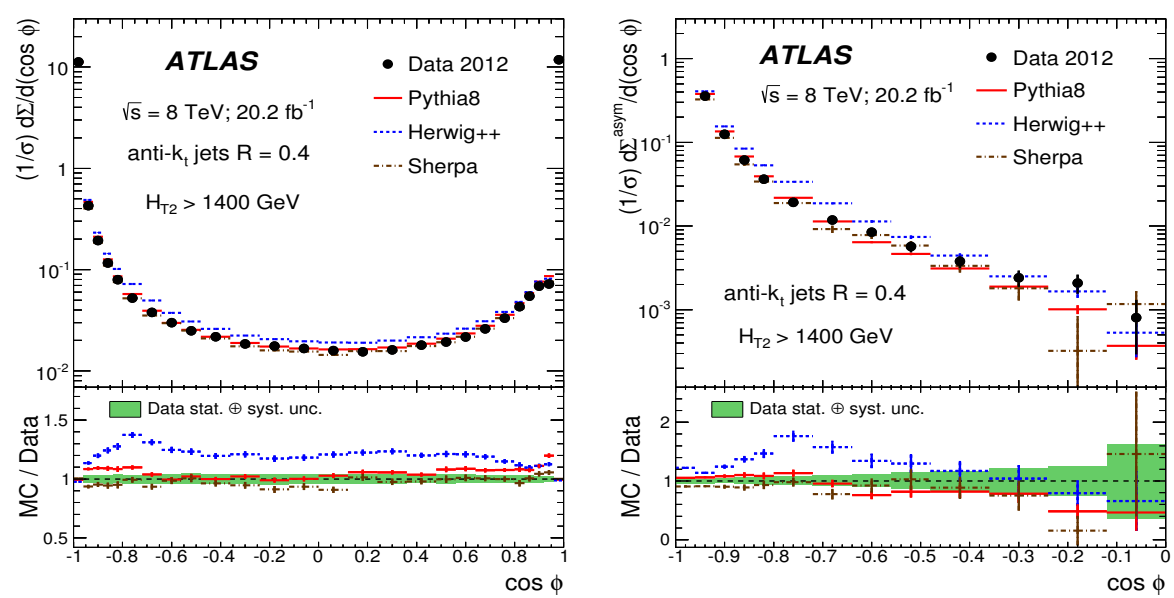

Fig. 1. Distributions of the TEEC (left) and ATEEC (right) for $H_{\mathrm{T} 2}>1400 \mathrm{GeV}$, along with Monte Carlo expectations from Pythia, Herwig++ and Sherpa. The error bars on the data points, as well as the green band in the lower panel, show the sum in quadrature of the statistical and systematic uncertainties.

For both the TEEC and ATEEC distributions, Pythia and Sherpa describe the data similarly and are the two Monte Carlo programs fitting the data best, while Herwig ++ shows significant discrepancies with the data.

\section{Experimental and theoretical uncertainties}

The experimental uncertainties for this measurement are summarised below

- Jet Energy Scale: The uncertainty on the jet energy measurement, estimated using 67 different nuisance parameters [10], amounts to approximately $2 \%$ on the TEEC distributions. 
- Jet Energy Resolution: Estimated by smearing the energy and momentum of each jet by a smearing factor accounting for the jet resolution. It amounts to about $1 \%$.

- Monte Carlo modelling: The correction for detector effects is performed using two different models, Pythia and Herwig++, and the difference between them is ascribed as the modelling uncertainty. This is the dominant experimental uncertainty in the analysis, being always below $5 \%$.

- Other experimental sources: This category comprises the correction for detector effects, jet angular resolution and jet cleaning uncertainties [5].

Figure 2 shows the relative systematic uncertainties mentioned above for the TEEC and ATEEC distributions in the higher $H_{\mathrm{T} 2}$ bin. The main sources of theoretical uncertainty affecting the measurement are the QCD scale uncertainty, which can reach up to $20 \%$ in the central TEEC region; the PDF uncertainty, of order $1 \%$ and the uncertainty on the corrections to the NLO predictions due to hadronisation and multiparton interactions, of order $1 \%$.
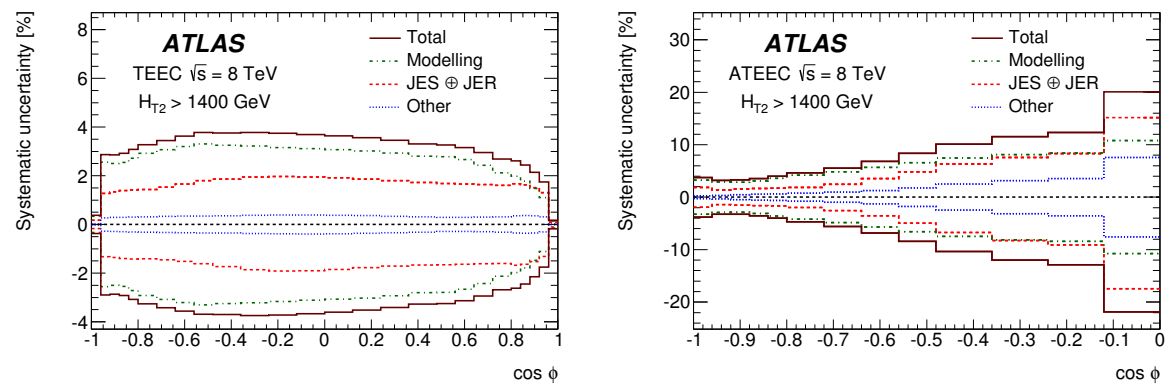

Fig. 2. Relative systematic uncertainties for the TEEC (left) and ATEEC (right) for $H_{\mathrm{T} 2}>1400$ $\mathrm{GeV}$. The total uncertainty is shown as a brown solid line.

\section{Determination of $\alpha_{\mathrm{s}}$}

The results presented in Section 2 are fitted to the NLO predictions by NLOJet++ [11] by minimising $\chi^{2}$ function with correlations among the sources of systematic uncertainties. The $\chi^{2}$ function can be written as

$$
\chi^{2}\left(\alpha_{\mathrm{s}}, \vec{\lambda}\right)=\sum_{\text {bins }} \frac{\left(x_{i}-F_{i}\left(\alpha_{\mathrm{s}}, \vec{\lambda}\right)\right)^{2}}{\Delta x_{i}^{2}+\Delta \xi_{i}^{2}}+\sum_{k} \lambda_{k}^{2} ; \quad F_{i}\left(\alpha_{\mathrm{s}}, \vec{\lambda}\right)=\psi_{i}\left(\alpha_{\mathrm{s}}\right)\left(1+\sum_{k} \lambda_{k} \sigma_{k}^{(i)}\right) .
$$

Here, $x_{i}$ are the data points and $\Delta x_{i}$ are its statistical uncertainties, while $\Delta \xi_{i}$ are the statistical uncertainties on the theoretical predictions. The relative value of the $k$-th systematic uncertainty in bin $i$ enters the fit as $\sigma_{k}^{(i)}$, while $\lambda_{k}$ are nuisance parameters used for evaluating the correlations between all systematic sources. The dependence of the observables with $\alpha_{\mathrm{s}}$ is parameterised using a second-order polynomial for each bin, denoted as $\psi_{i}\left(\alpha_{\mathrm{s}}\right)$. The values of $\alpha_{\mathrm{s}}$ obtained from fits to the 
ATEEC distributions are more precise than those obtained from the TEEC due to the fact that the scale uncertainties are smaller for ATEEC. From fits to the ATEEC distributions, the value of $\alpha_{s}\left(m_{Z}\right)$ is obtained. The resulting values are propagated to the measurement scale $Q=H_{\mathrm{T} 2} / 2$ using the NLO solution to the renormalisation group equation [5], and are shown in Fig. 3. The global fits to the TEEC and ATEEC data yield to

$$
\begin{aligned}
& \alpha_{\mathrm{s}}\left(m_{Z}\right)=0.1162 \pm 0.0011(\text { exp. })_{-0.0061}^{+0.0076}(\text { scale }) \pm 0.0018(\mathrm{PDF}) \pm 0.0003(\mathrm{NP}) \\
& \alpha_{\mathrm{s}}\left(m_{Z}\right)=0.1196 \pm 0.0013(\text { exp. })_{-0.0013}^{+0.0061}(\text { scale }) \pm 0.0017(\mathrm{PDF}) \pm 0.0004(\mathrm{NP})
\end{aligned}
$$

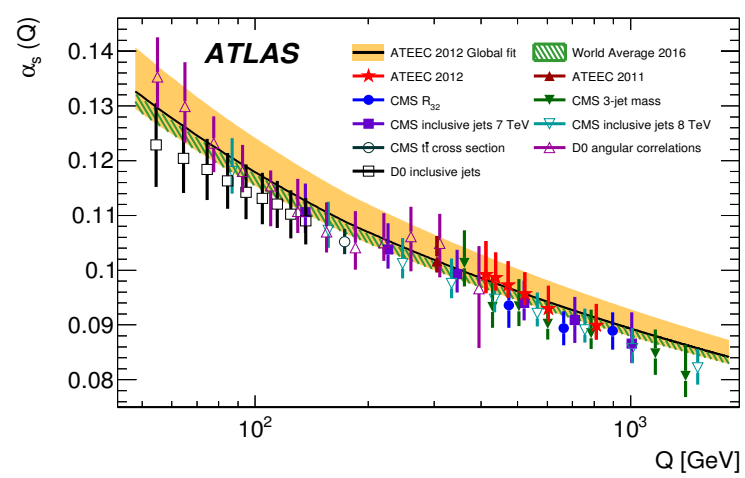

Fig. 3. Evolution of the strong coupling $\alpha_{\mathrm{S}}(Q)$ obtained from fits to the individual ATEEC distributions as a function of the energy scale. The results of individual fits are shown as red stars, while the result of the global fit is shown as a black solid line.

\section{Conclusion}

The measurement of the TEEC and ATEEC distributions has been presented. A good agreement with the expectations from Pythia and Sherpa is observed, while Herwig ++ is disfavoured. From fits to the TEEC and ATEEC distributions, the value of the strong coupling constant is obtained, as well as its evolution with the energy scale $Q=H_{\mathrm{T} 2} / 2$. The agreement of the observed running coupling with the results from previous experiments is excellent.

\section{References}

1. C. L. Basham et al. Phys. Rev. Lett. 41, 1585 (1978).

2. A. Ali et al. Phys. Lett. B 141, 447 (1984).

3. A. Ali et al. Phys. Rev. D 86, 114017 (2012).

4. ATLAS Collaboration. JINST 3 S08003 (2008).

5. ATLAS Collaboration. Eur. Phys. J. C 77, 872 (2017).

6. G. D'Agostini. Nucl. Instrum. Meth. A 362, 487 (1995).

7. T. Sjöstrand et al. Comp. Phys. Comm. 135, 238 (2001).

8. T. Gleisberg et al. JHEP 0902, 007 (2008).

9. M. Bahr et al. Eur. Phys. J. C 58, 639 (2008).

10. ATLAS Collaboration. Eur. Phys. J. C 75, 17 (2015).

11. Z. Nagy. Phys. Rev. Lett. 88, 122003 (2002). 